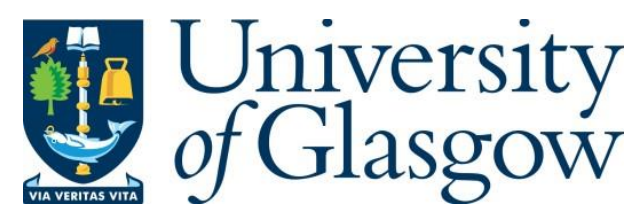

Poortvliet, R. K.E. et al. (2016) Risk stratification and treatment effect of statins in secondary cardiovascular prevention in old age: additive value of $\mathrm{N}$-terminal pro-B-type natriuretic peptide. European Journal of Preventive Cardiology, 23(10), pp. 1104-1113.

There may be differences between this version and the published version. You are advised to consult the publisher's version if you wish to cite from it.

http://eprints.gla.ac.uk/121337/

Deposited on: 22 September 2017

Enlighten - Research publications by members of the University of Glasgow http://eprints.gla.ac.uk 


\section{Risk Stratification and Treatment Effect of Statins in Secondary Cardiovascular Prevention in Old Age: Additive Value of NT-proBNP}

Rosalinde K.E. Poortvliet*1 \& Petra G. van Peet*1 ${ }^{*}$, Anton J.M. de Craen ${ }^{2}$, Bart J.A. Mertens ${ }^{3}$, Simon P. Mooijaart ${ }^{2,4,5}$, Liselotte W. Wijsman ${ }^{2,4}$, Yvonne M. Drewes ${ }^{1}$, Ian Ford ${ }^{6}$, Naveed Sattar $^{7}$, J. Wouter Jukema ${ }^{8}$, David J. Stott ${ }^{9}$, Wouter de Ruijter ${ }^{1}$, Jacobijn Gussekloo ${ }^{1}$

${ }^{1}$ Department of Public Health and Primary Care, Leiden University Medical Center, Leiden, the Netherlands

${ }^{2}$ Department of Gerontology and Geriatrics, Leiden University Medical Center, Leiden, the Netherlands

${ }^{3}$ Department of Medical Statistics, Leiden University Medical Center, Leiden, the Netherlands

${ }^{4}$ Netherlands Consortium for Healthy Ageing, Leiden, The Netherlands ${ }^{5}$ Institute for Evidence-Based Medicine in Old age, Leiden, the Netherlands ${ }^{6}$ Robertson Centre for Biostatistics, University of Glasgow, Glasgow, United Kingdom ${ }^{7}$ British Heart Foundation, Glasgow Cardiovascular Research Centre, University of Glasgow, Glasgow, United Kingdom

${ }^{8}$ Department of Cardiology, Leiden University Medical Center, Leiden, the Netherlands ${ }^{9}$ Academic Section of Geriatric Medicine, University of Glasgow-Faculty of Medicine, Glasgow Royal Infirmary, Glasgow, United Kingdom

*Authors had equal contribution

\section{Declaration of conflicting interests}

None declared 


\section{Funding}

The in-trial work in PROSPER was supported by an unrestricted investigator-initiated grant from Bristol-Myers Squibb, USA.

NT-proBNP measurements were funded by a grant from Biobanking and Biomolecular Research Infrastructure The Netherlands (grant number CP2011-33).

Role of the Funder/Sponsor: All funding sources were independent and had no influence on the design of this study; the collection, analyses, and interpretation of our data; the writing of this report; or the decision to submit the manuscript for publication.

Independence of researchers: All researchers worked independently from the funders.

\section{Corresponding author/request for reprints}

Rosalinde Poortvliet/Petra van Peet

Leiden University Medical Center

Dept. of Public Health and Primary Care (Postzone V0-P)

PO Box 9600

2300 RC Leiden

The Netherlands

r.k.e.poortvliet@lumc.nl/p.g.van_peet@lumc.nl

Telephone: 31-71-5268444

Fax: 31-71-5268259

Word count: abstract 253, full word count (including references, tables and figures) 5326, tables 3, figure 2, supplemental material 3 


\begin{abstract}
Background To date, no validated risk scores exist for prediction of recurrence risk or potential treatment effect for older people with a history of a cardiovascular event. Therefore, we assessed predictive values for recurrent cardiovascular disease (CVD), of models with age and sex, traditional cardiovascular risk markers, and 'SMART risk score', all with and without addition of N-terminal pro-B-type natriuretic peptide (NT-proBNP). Treatment effect of pravastatin was assessed across low and high risk groups identified by the best performing models.
\end{abstract}

Design and methods Post-hoc analysis in 2348 participants (age 70-82 years) with a history of CVD within the PROspective Study of Pravastatin in the Elderly at Risk (PROSPER) study. Composite endpoint was a recurrent cardiovascular event/cardiovascular mortality. Results The models with age and sex, traditional risk markers and SMART risk score had comparable predictive values (area under the curve (AUC) 0.58, 0.61 and 0.59, respectively). Addition of NT-proBNP to these models improved AUCs with $0.07\left(\mathrm{p}_{\text {diff }}=0.003\right), 0.05$ $\left(\mathrm{p}_{\text {diff }}=0.009\right)$ and $0.06\left(\mathrm{p}_{\text {diff }}<0.001\right)$, respectively. For the model with age, sex and NTproBNP, the hazard ratio for the composite endpoint in pravastatin users compared to placebo was 0.67 (95\%CI 0.49-0.90) for those in the highest third of predicted risk and $0.91(0.57-$ 1.46) in the lowest third, number needed to treat 12 and $115\left(\mathrm{p}_{\mathrm{diff}}=0.038\right)$ respectively. Conclusion In secondary cardiovascular prevention in old age addition of NT-proBNP improves prediction of recurrent CVD, cardiovascular mortality and treatment effect of pravastatin. A minimal model including age, sex and NT-proBNP predicts as good as complex risk models including NT-proBNP.

\title{
Keywords
}

Aged, Cardiovascular disease, Pro-brain natriuretic peptide, Hydroxymethylglutaryl-CoA reductase inhibitors, risk factors, secondary prevention 


\section{Introduction}

Persons with known cardiovascular disease are at high risk of recurrent events, and guidelines worldwide advise statins for secondary prevention, ${ }^{1-3}$ even in old age. ${ }^{4}$ Yet, prescription of secondary preventive treatment decreases with age. ${ }^{5,6}$ This might be caused by dilemmas regarding starting, continuing, or safely stopping preventive treatment, as physicians have to weigh postponed benefit versus current harm and priorities of care in old age. As many more patients are surviving their initial cardiovascular event, prediction of recurrent events becomes increasingly important. Ideally, the risk markers or risk models used, not only predict recurrence risk, but predict treatment effect as well. In secondary prevention in old age, traditional cardiovascular risk markers loose predictive value $\mathrm{e}^{7,8}$ and most risk scores are either too complex or only apply to restricted subgroups of hospitalized patients. ${ }^{9-12}$ To date, for the general older population, no risk scores for prediction of recurrence risk and/or treatment effect exist. Recently the SMART risk score was developed to predict recurrent cardiovascular events in a younger cohort of patients with a history of cardiovascular disease (mean age 60 years), ${ }^{13}$ but this risk score has not been validated in older age.

A new promising predictor of cardiovascular risk in old age is $\mathrm{N}$-terminal pro-brain natriuretic peptide (NT-proBNP), ${ }^{8,14}$ a polypeptide released in reaction to myocardial wall stress or ischemia. Addition of NT-proBNP to a model with the traditional cardiovascular risk markers or SMART risk score might improve predictive performance, especially in older patients.

Therefore, we first validated the SMART risk score in 1157 old subjects (mean age 75 years, placebo group) with a history of cardiovascular disease participating in the PROspective Study of Pravastatin in the Elderly at Risk (PROSPER). ${ }^{18}$ We compared the predictive value 
for recurrent cardiovascular events and mortality of the SMART risk score, with a model with traditional cardiovascular risk markers and with a minimal model including only age and sex. Second, we investigated whether addition of NT-proBNP to these prediction models could improve prediction. Third, we studied whether treatment effect of pravastatin was different across groups with low and high risk, calculated with the best performing models. 


\section{Methods}

\section{Study design}

Data in this study were obtained from the PROSPER study, a randomized, double-blind, placebo-controlled trial designed to investigate the effect of pravastatin in prevention of vascular events in older persons. Details of the design and outcome of PROSPER have been published elsewhere. ${ }^{18-20}$ Between December 1997 and May 1999, a total of 5804 individuals were screened and enrolled in Scotland, Ireland and the Netherlands. Men and women aged 70-82 years were recruited. A total of 2565 participants had a history of cardiovascular disease (including stable angina, intermittent claudication, stroke, transient ischemic attack, myocardial infarction and vascular surgery), and were included in the present study. (Flow chart provided as supplemental file 1)

Individuals with congestive heart failure (New York Heart Association functional class III and IV) or poor cognitive function (Mini-Mental State Examination score $<24$ points) were excluded from PROSPER. ${ }^{20}$ Participants were randomized into a group who received $40 \mathrm{mg}$ pravastatin a day and a control group receiving placebo and were followed 3.2 years on average. Throughout the study, all study personnel was unaware of the allocated study medication status of the participants. The institutional ethics review boards of all centres approved the protocol and all participants gave written informed consent. The protocol adhered to the principles of the Declaration of Helsinki.

\section{Traditional cardiovascular risk markers and SMART risk score variables}

During the pre-randomization visits, baseline participant characteristics were collected, ${ }^{19}$ including a detailed medical history with date(s) of last cardiovascular events, smoking status and current medication use. Participants weight, height and blood pressure were measured 
and fasting venous blood samples were taken including biobank samples. A history of diabetes was defined as a known diabetes mellitus or fasting blood glucose $>7 \mathrm{mmol} / \mathrm{L}$. Baseline serum creatinine levels were measured at central laboratories. Estimated glomerular filtration rate (eGFR) was estimated using the Modification of Diet in Renal Disease equation: ${ }^{21}$

$\mathrm{eGFR}=186 \times$ serum creatinine level $(\mathrm{mg} / \mathrm{dl})^{(-1.154)} \times \operatorname{age}^{(-0.203)} \times 0.742$ [if female].

Data of eGFR was missing for 5 included participants. High sensitivity C-reactive protein (hsCRP) levels were measured on stored $\mathrm{K}_{2}$ EDTA (at $-80^{\circ} \mathrm{C}$ ) baseline samples. ${ }^{22}$ Data of hsCRP was missing for 41 included participants. All laboratory analyses were conducted by technicians blind to the identity of samples and outcomes. Time since first cardiovascular event was calculated from the recorded date(s) of last cardiovascular event.

\section{NT-proBNP measurements}

Blood samples were taken at 6 months after baseline in EDTA tubes. ${ }^{20}$ The venous blood samples were stored in the biobank. From biobank samples NT-proBNP was determined using electrochemiluminescence immunoassay on a Roche Modulator E170. NT-proBNP measurements were missing for 167 participants due to technical problems.

\section{Outcomes}

For the present study the primary outcome of the trial was used: the combination of definite or suspect death from coronary heart disease, non-fatal myocardial infarction and fatal or non-fatal stroke. ${ }^{20}$ The PROSPER Endpoints Committee assessed all endpoints. The Endpoints Committee was blinded for study medication, and for plasma levels of NTproBNP. 


\section{Statistical analysis}

From the 2565 participants with a history of cardiovascular disease, participants with coronary events or who died in the first 6 months of the study $(n=50)$ and participants with missing NT-proBNP values at 6 months $(n=167)$ were excluded. Baseline summary characteristics are reported as median with interquartile range (IQR) for continuous variables and as numbers with percentage (\%) for categorical variables for all participants $(n=2348)$ and for participants on placebo and those on pravastatin separately. Follow-up for the outcomes was calculated from 6 months onward up to a maximum of 2.5 years.

\section{Calibration of the SMART risk score}

For calculation of the SMART risk score the SMART formula ${ }^{13}$ was used (Supplemental file 2). We used complete case analysis. Calibration of the SMART risk score for the PROSPER trial population was investigated by comparing the predicted versus observed cardiovascular disease risks. Participants taking placebo were divided into five categories of 2.5-year predicted risk, $<10 \%, 10$ to $<20 \%, 20$ to $<30 \%, 30$ to $<40 \%$, and $\geq 40 \%$. Within each category, predicted risk was compared to actual observed Kaplan-Meier cardiovascular disease free survival at 2.5 year follow-up (Supplemental file 3). In addition, the fitted regression coefficient (beta) was assessed in a Cox proportional hazard model fit, using only the linear prognostic score (A) as variable. ${ }^{23}$ The continuous predictive SMART prognostic risk score was multiplied with the calculated regression coefficient to recalibrate the SMART risk score for the PROSPER population, as the calibrated regression coefficient significantly differed from $1(0.466, \mathrm{p}<0.001)$.

Risk prediction with three models in the placebo group 
The 2.5-year cardiovascular disease risk (\%) was predicted for all participants using a Cox proportional hazards models (complete case analysis) fit based on 1) age and sex (minimal model); 2) age, sex, smoking, systolic blood pressure, high density lipoprotein and total cholesterol, history of diabetes, history of hypertension, history of myocardial infarction, history of stroke/transient ischaemic attack and history of surgery for peripheral artery disease (all as assessed at baseline; traditional model); and 3) recalibrated SMART risk score (SMART model). Using the continuous predicted risks from the three models, area under the curves (AUCs) and receiver operating characteristic (ROC) curves with p-values (level of significance 5\%) and 95\% confidence intervals for difference were calculated.

\section{Additional value of NT-proBNP in the placebo group} NT-proBNP was non-normally distributed and therefore log transformed. Cox proportional hazards models for the occurrence of the primary endpoint were fitted based on three additional models including 1) minimal model plus NT-proBNP; 2) traditional model plus NT-proBNP; and 3) SMART model plus NT-proBNP. AUCs and ROC curves were calculated and compared to the reference models without NT-proBNP (STATA 12.1). Cross validation using the Jack-knife method, was used for comparison of optimism-corrected estimates. $^{24}$

\section{Net Reclassification Improvement}

We calculated the category-less Net Reclassification Improvement (NRI) for the primary endpoint with logistic regression, comparing the three models with NT-proBNP models to the reference models without NT-proBNP. ${ }^{25,26}$ 
Predicted risk for the primary endpoint was calculated for all participants using the regression coefficients from the models developed in the placebo group. The treatment effect of pravastatin according to the thirds of predicted risk for the primary endpoint of the three models including NT-proBNP was assessed in three ways. First, the presence of multiplicative interaction was tested by adding the interaction term 'treatment $\mathrm{x}$ thirds of predicted risk' in the Cox model. Second, per third of predicted risk, the absolute numbers of events in the pravastatin group and the placebo groups were calculated and the absolute risk reduction (ARR) by pravastatin was calculated using the life-table method. Differences in ARR between the thirds of predicted risk, were tested using a $z$-test. Numbers needed to treat (NNT) were calculated over 2.5 years based on the difference in cumulative proportion surviving in the pravastatin and placebo groups. Finally, the hazard ratio (HR) for the occurrence of cardiovascular events in the pravastatin group versus placebo group was calculated using the Cox proportional hazard model per third of predicted risk. 


\section{Results}

Table 1 presents the baseline characteristics for the participants. Of the 2348 participants $57 \%$ $(n=1334)$ were men, $73 \%(n=1713)$ had a history of cardiac disease, $25 \%(n=594)$ had a history of cerebrovascular disease and $17 \%(n=408)$ had a history of peripheral disease. The median NT-proBNP level was 176 ng/L (IQR 96-359).

\section{Traditional cardiovascular risk markers and SMART risk score}

During the maximum follow-up of 2.5 years, $16 \%(n=187)$ of participants in the placebo group ( $\mathrm{n}=1157)$ developed a cardiovascular event or died of cardiovascular disease (primary endpoint). During follow up 147(12.7\%) patients developed a coronary event and 43(3.7\%) a fatal or non-fatal stroke.

We calculated AUCs and created ROC curves for the minimal model, the traditional model and the SMART model, with the primary endpoint at 2.5-year (Figure 1). The three models had similar AUCs: 0.58 (95\% CI 0.54-0.63) for the minimal model; 0.61 (95\%CI 0.57-0.66) for the traditional model; and 0.59 (95\% CI 0.54-0.63) for the SMART model (Table 2).

\section{Addition of NT-proBNP}

Figure 1 shows that the addition of NT-proBNP improved the AUC of all three models similarly. Addition of NT-proBNP to the minimal model increased the AUC from 0.58 to $0.65(95 \%$ CI $0.6-0.70), \Delta 0.07, p$ for difference $\left(\mathrm{p}_{\text {diff }}\right)=0.003$. The increase in AUC was similar for both the traditional and the SMART model $\left(\Delta 0.05, \mathrm{p}_{\text {diff }}=0.009\right.$ and $\Delta 0.06$, $\mathrm{p}_{\text {diff }}<0.001$, respectively) (Table 2$)$. 
The minimal model with addition of NT-proBNP performed similarly to the traditional model with addition of NT-proBNP ( $\mathrm{p}_{\text {diff }}=0.26$ ) as well as to the SMART model plus NT-proBNP $\left(\mathrm{p}_{\text {diff }}=0.87\right)$.

Cross validation of the minimal model led to an AUC of 0.56 (95\% CI 0.52-0.61) and for the minimal model with addition of NT-proBNP to an AUC of 0.64 (95\% CI 0.60-0.69). The difference between these two cross validated AUCs was $0.08(\mathrm{p}=0.0016)$. Cross validation of the other models showed similar results (data not shown).

\section{$N R I$}

The category-less NRI with addition of NT-proBNP to the minimal model was $41 \%$ ( $<<0.001,57.2 \%$ of participants reclassified up minus $42.8 \%$ reclassified down in the group that experienced the endpoint, plus $63.2 \%$ reclassified down minus $36.8 \%$ reclassified up in the group that did not experience the endpoint). The category-less NRI with addition of NTproBNP to the traditional model was $39 \%$ ( $\mathrm{p}<0.001$ ). Addition of NT-proBNP to the SMART model had an NRI of $25 \%(\mathrm{p}=0.002)$ (Table 2$)$.

\section{Treatment effect}

Overall, in the 2348 participants with a history of cardiovascular disease within the PROSPER study population, the ARR by pravastatin treatment was $3.6 \%$ for 2.5 year. After, the 2.5-year HR for the development of the primary endpoint was 0.77 (95\% CI $0.62-0.95)$ in the pravastatin group compared to the placebo group.

We divided participants according to thirds of predicted risk. Multiplicative interaction between treatment and thirds of predicted risks of all models was not significant (all $\mathrm{p}>0.1$ ). 
Table 3 shows the treatment effect (2.5-year) of pravastatin according to thirds of predicted risk of cardiovascular disease and mortality for three risk models, all with NT-proBNP, including number of events (primary endpoint), ARR and HR. The ARR in primary endpoint with 2.5-year pravastatin treatment in the low predicted risk group of the minimal model plus NT-proBNP was $0.87 \%$ (95\% CI -3.2-4.9) and in the high predicted risk group $8.2 \%$ (95\% CI 2.6-13.9), difference $=7.4 \%\left(95 \%\right.$ CI 0.43-14.3, $\left.\mathrm{p}_{\text {diff }}=0.038\right)$. (Figure 2$)$ In this model, participants with the highest predicted risk (highest third) and pravastatin treatment had a HR of 0.67 (95\% CI 0.49-0.90) for the development of the primary endpoint compared to those on placebo. The NNT during 2.5 years with pravastatin was 12 (95\% CI 7-38). HR for participants in the lowest third of predicted risk was 0.91 (95\% CI 0.57-1.46), with a NNT of $115(95 \%$ CI $20-\infty)$. 


\section{Discussion}

This study shows that the predictive value of traditional cardiovascular risk markers and the (recalibrated) SMART risk score is poor in older people with a history of cardiovascular disease, and comparable to prediction with a model including only age and sex. Addition of NT-proBNP, however, improved prediction of recurrent cardiovascular disease and mortality. We observed that a model with age, sex and NT-proBNP predicts as good as more complex risk models including NT-proBNP. Moreover in high risk individuals as identified by age, sex plus NT-proBNP level, NNT for 2.5-year pravastatin treatment was 12, whereas, in patients with a low predicted risk in this model, NNT was 115 . As many more patients are surviving their initial cardiovascular event, prediction and prevention of recurrent events becomes increasingly important. According to this study NT-proBNP is a promising risk predictor in old age.

\section{Comparison with the literature}

The combination of prediction of recurrent events and treatment effect has seldom been examined in secondary cardiovascular prevention. Our findings contrast with the findings in the CORONA and Heart Protection Study in patients with chronic heart failure, where the benefit of rosuvastatin was higher in the low NT-proBNP group. However, this relationship might have been modified by other patient characteristics in this specific population of ischemic heart failure patients. ${ }^{27}$

Previously, Sattar et al. have investigated within the entire PROSPER study population whether hsCRP could predict treatment effect and they observed that hsCRP did not predict response to statin therapy. ${ }^{22}$ In contrast, Drewes et al. found a positive relation of homocysteine levels with treatment effect. ${ }^{28}$ However, physicians are perhaps more inclined 
to determine serological biomarkers that have a direct association with cardiac strain such as NT-proBNP.

With regard to prediction of recurrent events, the predictive value of NT-proBNP has been described in primary as well as in secondary prevention, ${ }^{14}$ even in very old age ${ }^{8,15,17}$ and in persons with ${ }^{29}$ and without clinical heart failure. ${ }^{30}$ In the literature, addition of NT-proBNP to traditional cardiovascular risk markers results in an improvement of the AUC ranging from 0.01-0,1. ${ }^{8,14,31}$ The HOPE study findings ${ }^{31}$ showed that of all biomarkers added to traditional risk markers in secondary prevention, NT-proBNP was the strongest (increase in AUC 0.05 as compared to traditional risk markers, $\mathrm{p}<0.001)$. This is consistent with the present study in a secondary prevention population. The SMART risk score, which includes hsCRP, was not superior to the model including age and sex. This might be explained by the decreasing predictive value of hsCRP with age, ${ }^{32,33}$ as our study population was older by around 15 years on average, than the population in which the original SMART risk score was developed. Also, even if the true risks are the same in both populations, shrinkage can be expected when a prediction model is validated in a different population.

\section{Implications for clinical practice and future research}

In our cohort of older persons the SMART risk score had to be recalibrated as it overestimated actual risk for recurrent cardiovascular disease and cardiovascular mortality, especially in persons assigned to the high risk category. Physicians should be aware of the derivation cohort characteristics, before applying new risk scores to their patients.

Our result suggest than in secondary cardiovascular prevention in old age, measuring NTproBNP helps physicians better estimate recurrence risk. An additional advantage might be 
that a high NT-proBNP level prompts clinicians to actively search for signs and symptoms of heart failure or (paroxysmal) atrial fibrillation. ${ }^{34}$ More complex models are not required as predictive value was the same as in a model with age and sex only. However this requires validation and subsequent evaluation of clinical impact, especially regarding treatment effect, before it can be implemented. Nevertheless, the wide availability of NT pro-BNP assays in routine laboratories means clinical translation of our findings is ultimately possible. When in doubt whether or not to start, stop or continue secondary preventive treatment with statins in old age, measurement of NT-proBNP can help clinicians and patients to estimate future risk and expected treatment effect. A simple risk prediction model with only a few risk markers (age, sex and NT-proBNP) is easy to use in clinical practice and seems appropriate.

Future etiological studies are necessary to establish the possible causal associations between NT-proBNP and cardiovascular and cerebrovascular morbidity and mortality.

\section{Strengths and Limitations}

To analyse NT-proBNP levels in the large well-defined secondary prevention population within the PROSPER study population, and to calculate treatment effect accordingly, was a tempting opportunity, since placebo controlled RCT's concerning treatment effect of statins are ethically impossible to perform in the present era.

PROSPER is a randomised controlled trial, therefore, the participants were selected using more strict criteria than in a cohort study, like the SMART study. A potential limitation in this respect is the exclusion of persons with clinical heart failure or poor cognitive function. The observed risks could have been influenced. NT-proBNP was measured at 6 months, not at baseline due to limited plasma availability in the latter. Therefore, follow-up was calculated from 6 months onward. Pravastatin treatment had no effect on NT-proBNP levels 
in the first 6 months, which is in line with previous studies. ${ }^{35}$ Since NT-proBNP was measured at 6 months from baseline, we had to exclude participants that already died or experienced a cardiac event in the first 6 months of the study ( $2 \%$ of the study population). As these participants are likely to be high risk individuals in the models, exclusion may have led to an underestimation of the true magnitude of predictive value of the models. It is also a limitation that cholesterol, CRP and eGFR were measured at baseline and not measured at six months (like NT-proBNP), as values of cholesterol, CRP and eGFR might have changed in this 6 month period. However, the use of cholesterol levels measured at month six would be inappropriate since the pravastatin treatment changes the cholesterol level and would therefore lead to incorrect results in the evaluation of the treatment effect.

Finally, the relatively low AUCs might be considered as a limitation. However, an AUC between 0.65 and 0.70 is common in studies in older populations. ${ }^{8,36}$

\section{Conclusions}

Due to increased survival following an acute cardiovascular event, also in old age, adequate prediction of recurrent events is becoming increasingly important. According to this study NT-proBNP is a promising risk predictor. Addition of NT-proBNP to (traditional) risk models improves prediction in old age. Moreover, a minimal model with only age, sex and NT-proBNP is as good as complex risk models including NT-proBNP. 


\section{Declaration of conflicting interests}

None declared

\section{Funding}

The in-trial work in PROSPER was supported by an unrestricted investigator-initiated grant from Bristol-Myers Squibb, USA.

NT-proBNP measurements were funded by a grant from Biobanking and Biomolecular Research Infrastructure The Netherlands (grant number CP2011-33).

Role of the Funder/Sponsor: All funding sources were independent and had no influence on the design of this study; the collection, analyses, and interpretation of our data; the writing of this report; or the decision to submit the manuscript for publication.

Independence of researchers: All researchers worked independently from the funders. 


\section{References}

1. National Guideline Clearinghouse N. European guidelines on cardiovascular disease prevention in clinical practice (version 2012).

http://www.guideline.gov/content.aspx?f=rss\&id=39354 (7/14/2014).

2. National Guideline Clearinghouse N. 2012 ACCF/AHA/ACP/AATS/PCNA/SCAI/STS guideline for the diagnosis and management of patients with stable ischemic heart disease. http://www.guideline.gov/content.aspx?id=39380 (7/14/2014).

3. National Guideline Clearinghouse N. Primary and secondary prevention of cardiovascular disease: antithrombotic therapy and prevention of thrombosis, 9th ed: American College of Chest Physicians evidence-based clinical practice guidelines. http://www.guideline.gov/content.aspx?f=rss\&id=35273 (7/14/2014).

4. Afilalo J, Duque G, Steele R, Jukema JW, de Craen AJ, Eisenberg MJ. Statins for secondary prevention in elderly patients: a hierarchical bayesian meta-analysis. $\mathrm{J} \mathrm{Am}$ Coll Cardiol 2008;51:37-45.

5. Koopman C, Vaartjes I, Heintjes EM, Spiering W, van Dis I, Herings RM, Bots ML. Persisting gender differences and attenuating age differences in cardiovascular drug use for prevention and treatment of coronary heart disease, 1998-2010. Eur Heart J 2013;34:3198-3205.

6. Ko DT, Mamdani M, Alter DA. Lipid-lowering therapy with statins in high-risk elderly patients: the treatment-risk paradox. JAMA 2004;291:1864-1870.

7. Uthoff H, Staub D, Socrates T, Meyerhans A, Bundi B, Schmid HP, Frauchiger B. PROCAM-, FRAMINGHAM-, SCORE- and SMART-risk score for predicting cardiovascular morbidity and mortality in patients with overt atherosclerosis. Vasa 2010;39:325-333.

8. van Peet PG, Drewes YM, de Craen AJ, Gussekloo J, de Ruijter W. NT-proBNP best predictor of cardiovascular events and cardiovascular mortality in secondary prevention in very old age: the Leiden 85-plus Study. PLoS One 2013;8:e81400.

9. Thompson DD, Murray GD, Dennis M, Sudlow CL, Whiteley WN. Formal and informal prediction of recurrent stroke and myocardial infarction after stroke: a systematic review and evaluation of clinical prediction models in a new cohort. $B M C$ Med 2014;12:58.

10. Fox KA, Dabbous OH, Goldberg RJ, Pieper KS, Eagle KA, Van de Werf F, Avezum A, Goodman SG, Flather MD, Anderson FA, Jr., Granger CB. Prediction of risk of death and myocardial infarction in the six months after presentation with acute coronary syndrome: prospective multinational observational study (GRACE). BMJ 2006;333:1091.

11. Lafitte M, Pucheu Y, Latry K, Dijos M, Casassus E, Couffinhal T. Predictors of cardiovascular prognosis in patients receiving optimized secondary prevention measures after acute coronary syndrome. Eur J Prev Cardiol 2013;20:283-290. 
12. Riedel M, Lafitte M, Pucheu Y, Latry K, Couffinhal T. Prognostic value of highsensitivity C-reactive protein in a population of post-acute coronary syndrome patients receiving optimal medical treatment. Eur J Prev Cardiol 2012;19:1128-1137.

13. Dorresteijn JA, Visseren FL, Wassink AM, Gondrie MJ, Steyerberg EW, Ridker PM, Cook NR, van der Graaf Y. Development and validation of a prediction rule for recurrent vascular events based on a cohort study of patients with arterial disease: the SMART risk score. Heart 2013;99:866-872.

14. Di Angelantonio E, Chowdhury R, Sarwar N, Ray KK, Gobin R, Saleheen D, Thompson A, Gudnason V, Sattar N, Danesh J. B-type natriuretic peptides and cardiovascular risk: systematic review and meta-analysis of 40 prospective studies. Circulation 2009;120:2177-2187.

15. van Peet PG, de Craen AJ, Gussekloo J, de Ruijter W. Plasma NT-proBNP as predictor of change in functional status, cardiovascular morbidity and mortality in the oldest old: the Leiden 85-plus study. Age (Dordr ) 2014;36:9660.

16. Wannamethee SG, Welsh P, Lowe GD, Gudnason V, Di Angelantonio E, Lennon L, Rumley A, Whincup PH, Sattar N. N-terminal pro-brain natriuretic Peptide is a more useful predictor of cardiovascular disease risk than C-reactive protein in older men with and without pre-existing cardiovascular disease. J Am Coll Cardiol 2011;58:56-64.

17. Vaes B, de Ruijter W, Degryse J, Westendorp RG, Gussekloo J. Clinical relevance of a raised plasma $\mathrm{N}$-terminal pro-brain natriuretic peptide level in a population-based cohort of nonagenarians. J Am Geriatr Soc 2009;57:823-829.

18. Shepherd J, Blauw GJ, Murphy MB, Bollen EL, Buckley BM, Cobbe SM, Ford I, Gaw A, Hyland M, Jukema JW, Kamper AM, Macfarlane PW, Meinders AE, Norrie J, Packard CJ, Perry IJ, Stott DJ, Sweeney BJ, Twomey C, Westendorp RG. Pravastatin in elderly individuals at risk of vascular disease (PROSPER): a randomised controlled trial. Lancet 2002;360:1623-1630.

19. Ford I, Blauw GJ, Murphy MB, Shepherd J, Cobbe SM, Bollen EL, Buckley BM, Jukema JW, Hyland M, Gaw A, Lagaay AM, Perry IJ, Macfarlane P, Norrie J, Meinders AE, Sweeney BJ, Packard CJ, Westendorp RG, Twomey C, Stott DJ. A Prospective Study of Pravastatin in the Elderly at Risk (PROSPER): Screening Experience and Baseline Characteristics. Curr Control Trials Cardiovasc Med 2002;3:8.

20. Shepherd J, Blauw GJ, Murphy MB, Cobbe SM, Bollen EL, Buckley BM, Ford I, Jukema JW, Hyland M, Gaw A, Lagaay AM, Perry IJ, Macfarlane PW, Meinders AE, Sweeney BJ, Packard CJ, Westendorp RG, Twomey C, Stott DJ. The design of a prospective study of Pravastatin in the Elderly at Risk (PROSPER). PROSPER Study Group. PROspective Study of Pravastatin in the Elderly at Risk. Am J Cardiol 1999;84:1192-1197.

21. Levey AS, Bosch JP, Lewis JB, Greene T, Rogers N, Roth D. A more accurate method to estimate glomerular filtration rate from serum creatinine: a new prediction equation. Modification of Diet in Renal Disease Study Group. Ann Intern Med 1999;130:461-470. 
22. Sattar N, Murray HM, McConnachie A, Blauw GJ, Bollen EL, Buckley BM, Cobbe SM, Ford I, Gaw A, Hyland M, Jukema JW, Kamper AM, Macfarlane PW, Murphy MB, Packard CJ, Perry IJ, Stott DJ, Sweeney BJ, Twomey C, Westendorp RG, Shepherd J. C-reactive protein and prediction of coronary heart disease and global vascular events in the Prospective Study of Pravastatin in the Elderly at Risk (PROSPER). Circulation 2007;115:981-989.

23. Cox DR. Two further applications of a model for binary regression. Biometrika 1958;45:562-565.

24. Van Houwelingen JC, Le Cessie S. Predictive value of statistical models. Stat Med 1990;9:1303-1325.

25. Pencina MJ, d'Agostino RB, Vasan RS. Statistical methods for assessment of added usefulness of new biomarkers. Clin Chem Lab Med 2010;48:1703-1711.

26. Pencina MJ, D'Agostino RB, Sr., Steyerberg EW. Extensions of net reclassification improvement calculations to measure usefulness of new biomarkers. Stat Med 2011;30:11-21.

27. Daniels LB, Barrett-Connor E. Can natriuretic peptides help identify heart failure patients for whom statins are beneficial? J Am Coll Cardiol 2009;54:1860-1861.

28. Drewes YM, Poortvliet RK, Blom JW, de Ruijter W, Westendorp RG, Stott DJ, Blom HJ, Ford I, Sattar N, Wouter JJ, Assendelft WJ, de Craen AJ, Gussekloo J. Homocysteine levels and treatment effect in the prospective study of pravastatin in the elderly at risk. J Am Geriatr Soc 2014;62:213-221.

29. Don-Wauchope AC, Santaguida PL, Oremus M, McKelvie R, Ali U, Brown JA, Bustamam A, Sohel N, Hill SA, Booth RA, Balion C, Raina P. Incremental predictive value of natriuretic peptides for prognosis in the chronic stable heart failure population: a systematic review. Heart Fail Rev 2014;19:521-540.

30. Wang TJ, Larson MG, Levy D, Benjamin EJ, Leip EP, Omland T, Wolf PA, Vasan RS. Plasma natriuretic peptide levels and the risk of cardiovascular events and death. $N$ Engl J Med 2004;350:655-663.

31. Blankenberg S, McQueen MJ, Smieja M, Pogue J, Balion C, Lonn E, Rupprecht HJ, Bickel C, Tiret L, Cambien F, Gerstein H, Munzel T, Yusuf S. Comparative impact of multiple biomarkers and N-Terminal pro-brain natriuretic peptide in the context of conventional risk factors for the prediction of recurrent cardiovascular events in the Heart Outcomes Prevention Evaluation (HOPE) Study. Circulation 2006;114:201-208.

32. Olsen MH, Hansen TW, Christensen MK, Gustafsson F, Rasmussen S, Wachtell K, Ibsen H, Torp-Pedersen C, Hildebrandt PR. Cardiovascular risk prediction by Nterminal pro brain natriuretic peptide and high sensitivity $\mathrm{C}$-reactive protein is affected by age and sex. J Hypertens 2008;26:26-34.

33. Wannamethee SG, Welsh P, Lowe GD, Gudnason V, Di AE, Lennon L, Rumley A, Whincup PH, Sattar N. N-terminal pro-brain natriuretic Peptide is a more useful predictor of cardiovascular disease risk than $\mathrm{C}$-reactive protein in older men with and without pre-existing cardiovascular disease. J Am Coll Cardiol 2011;58:56-64. 
34. Patton KK, Ellinor PT, Heckbert SR, Christenson RH, DeFilippi C, Gottdiener JS, Kronmal RA. N-terminal pro-B-type natriuretic peptide is a major predictor of the development of atrial fibrillation: the Cardiovascular Health Study. Circulation 2009;120:1768-1774.

35. Welsh P, Doolin O, Willeit P, Packard C, Macfarlane P, Cobbe S, Gudnason V, Di Angelantonio E, Ford I, Sattar N. N-terminal pro-B-type natriuretic peptide and the prediction of primary cardiovascular events: results from 15-year follow-up of WOSCOPS. Eur Heart J 2013;34:443-450.

36. de Ruijter W, Westendorp RG, Assendelft WJ, den Elzen WP, de Craen AJ, Le Cessie $\mathrm{S}$, Gussekloo J. Use of Framingham risk score and new biomarkers to predict cardiovascular mortality in older people: population based observational cohort study. BMJ 2009;338:a3083. 


\section{Figure legends}

Figure 1. Receiver operating characteristic curves for three models without NT-proBNP (dotted lines) and with NT-proBNP (black lines) for cardiovascular events and cardiovascular mortality. Model with age and sex (left, minimal model) model with traditional risk markers (middle, traditional model) and model with SMART risk score (right, SMART model) (p $\Delta$ $0.003,0.003$ and $<0.001$, respectively)

Figure 2. Absolute risk reduction (ARR) and number needed to treat to benefit (NNTB) and number needed to harm $(\mathrm{NNTH})$ with pravastatin for 2.5 years, according to tertiles of predicted risk, $\mathrm{p}$-value of difference between lowest and highest predicted risk group for NNTB, estimated using $z$-test 
Table 1 Baseline characteristics of the participants stratified for placebo and pravastatin group

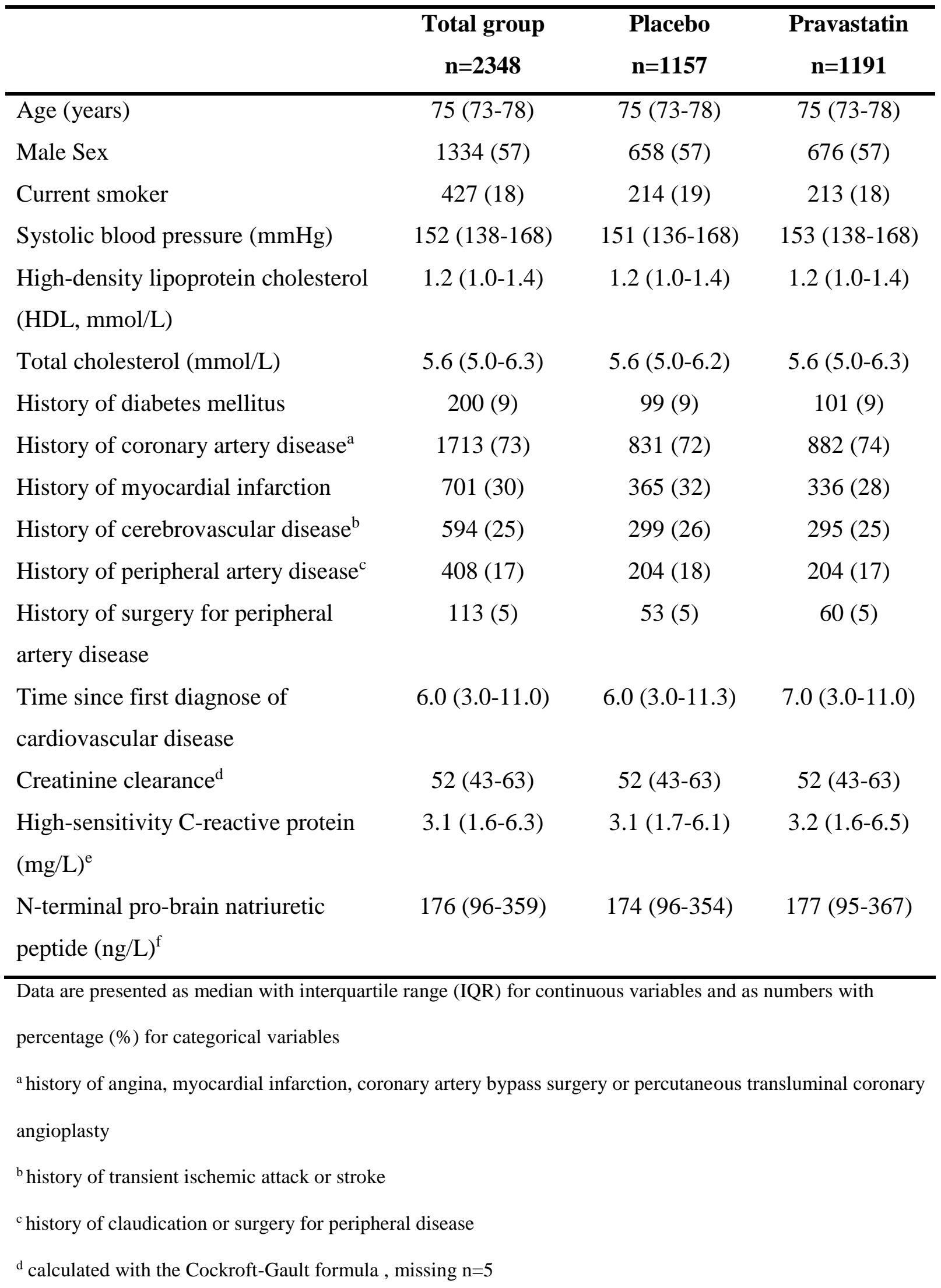


e missing $n=41$

${ }^{\mathrm{f}}$ measured at 6 months after study entrance 
Table 2 Absolute number of events in tertiles of predicted risk of the different models, with area under the curve (AUC), delta AUC ( $\triangle \mathrm{AUC}$ ) with addition of NT-proBNP, and category free net reclassification improvement (NRI) for the primary endpoint in the placebo group $(n=1157)$

\begin{tabular}{|c|c|c|c|c|c|c|c|c|}
\hline \multirow[t]{2}{*}{ Risk models } & \multicolumn{3}{|c|}{$\begin{array}{c}\text { Absolute numbers of events in tertiles of } \\
\text { risk }\end{array}$} & \multirow[t]{2}{*}{$\operatorname{AUC}(95 \% \mathrm{CI})$} & \multirow[t]{2}{*}{$\triangle \mathrm{AUC}$} & \multirow[t]{2}{*}{$\mathbf{p} \Delta$} & \multirow[t]{2}{*}{ NRI (\%) } & \multirow[t]{2}{*}{ p value } \\
\hline & Low & Medium & High & & & & & \\
\hline Minimal model & $43(11.2)$ & $68(17.8)$ & $76(19.4)$ & $0.58(0.54-0.63)$ & & & & \\
\hline $\begin{array}{l}\text { Minimal model } \\
\text { plus NT-proBNP }\end{array}$ & $37(9.5)$ & $54(13.7)$ & $96(25.7)$ & $0.66(0.61-0.70)$ & 0.07 & 0.0026 & 41 & $<0.001$ \\
\hline Traditional model & $42(11.0)$ & $56(14.6)$ & $89(22.7)$ & $0.61(0.57-0.65)$ & & & & \\
\hline $\begin{array}{l}\text { Traditional model } \\
\text { plus NT-proBNP }\end{array}$ & $35(9.0)$ & $54(13.7)$ & $98(26.1)$ & $0.66(0.62-0.70)$ & 0.05 & 0.0091 & 39 & $<0.001$ \\
\hline SMART model & $60(15.8)$ & $60(16.0)$ & $65(16.9)$ & $0.59(0.54-0.63)$ & & & & \\
\hline $\begin{array}{l}\text { SMART model } \\
\text { plus NT-proBNP }\end{array}$ & $53(14.4)$ & $65(16.0)$ & $67(18.3)$ & $0.65(0.61-0.70)$ & 0.06 & 0.0006 & 25 & 0.002 \\
\hline
\end{tabular}


Table 3 Treatment effect after 2.5-year of treatment with pravastatin according to tertiles of predicted risk of cardiovascular disease and mortality for three risk models including NT-proBNP

\begin{tabular}{lcccc}
\hline & $\begin{array}{c}\text { Events in pravastatin } \\
\text { group }\end{array}$ & $\begin{array}{c}\text { Events in placebo } \\
\text { group }\end{array}$ & ARR (95\% CI) \\
\hline Minimal model plus NT-proBNP & $34(8.7)$ & $37(9.5)$ & $0.87(-3.2-4.9)$ & $0.91(0.57-1.46)$ \\
Low & $42(10.8)$ & $54(13.7)$ & $2.9(-1.8-7.5)$ & $0.78(0.52-1.17)$ \\
Medium & $74(18.0)$ & $96(25.7)$ & $8.2(2.6-13.9)$ & $0.67(0.49-0.90)$ \\
High & & & & \\
Traditional model plus NT-proBNP & $26(6.6)$ & $35(9.0)$ & $2.5(-1.28-6.33)$ & $0.73(0.44-1.20)$ \\
Low & $45(11.6)$ & $54(13.7)$ & $2.0(-2.73-6.77)$ & $0.83(0.56-1.24)$ \\
Medium & $79(19.4)$ & $98(26.1)$ & $7.2(1.18-13.29)$ & $0.71(0.53-0.95)$ \\
High & & & $3.0(-1.86-7.78)$ & $0.78(0.52-1.15)$ \\
SMART model plus NT-proBNP & $45(11.3)$ & $53(14.4)$ & $0.72(0.49-1.06)$ \\
Low & $42(11.6)$ & $65(16.0)$ & $4.5(-0.43-9.45)$ & $0.78(0.55-1.11)$ \\
Medium & $60(14.9)$ & $67(18.3)$ & $4.0(-1.45-9.43)$
\end{tabular}

ARR, absolute risk reduction

HR, hazard ratio 
Figure 1
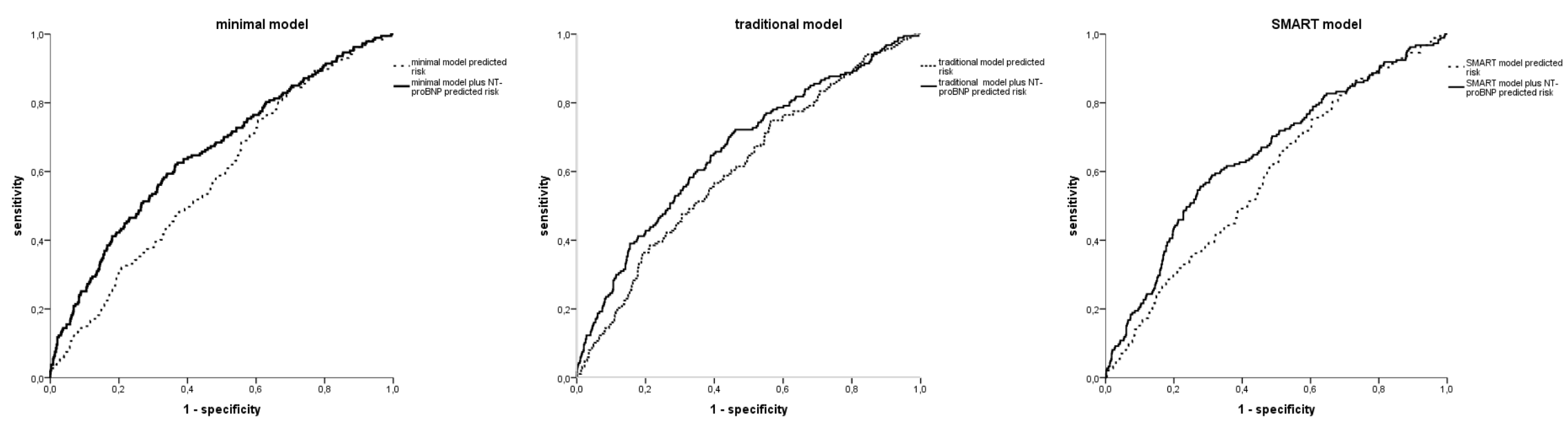
Figure 2

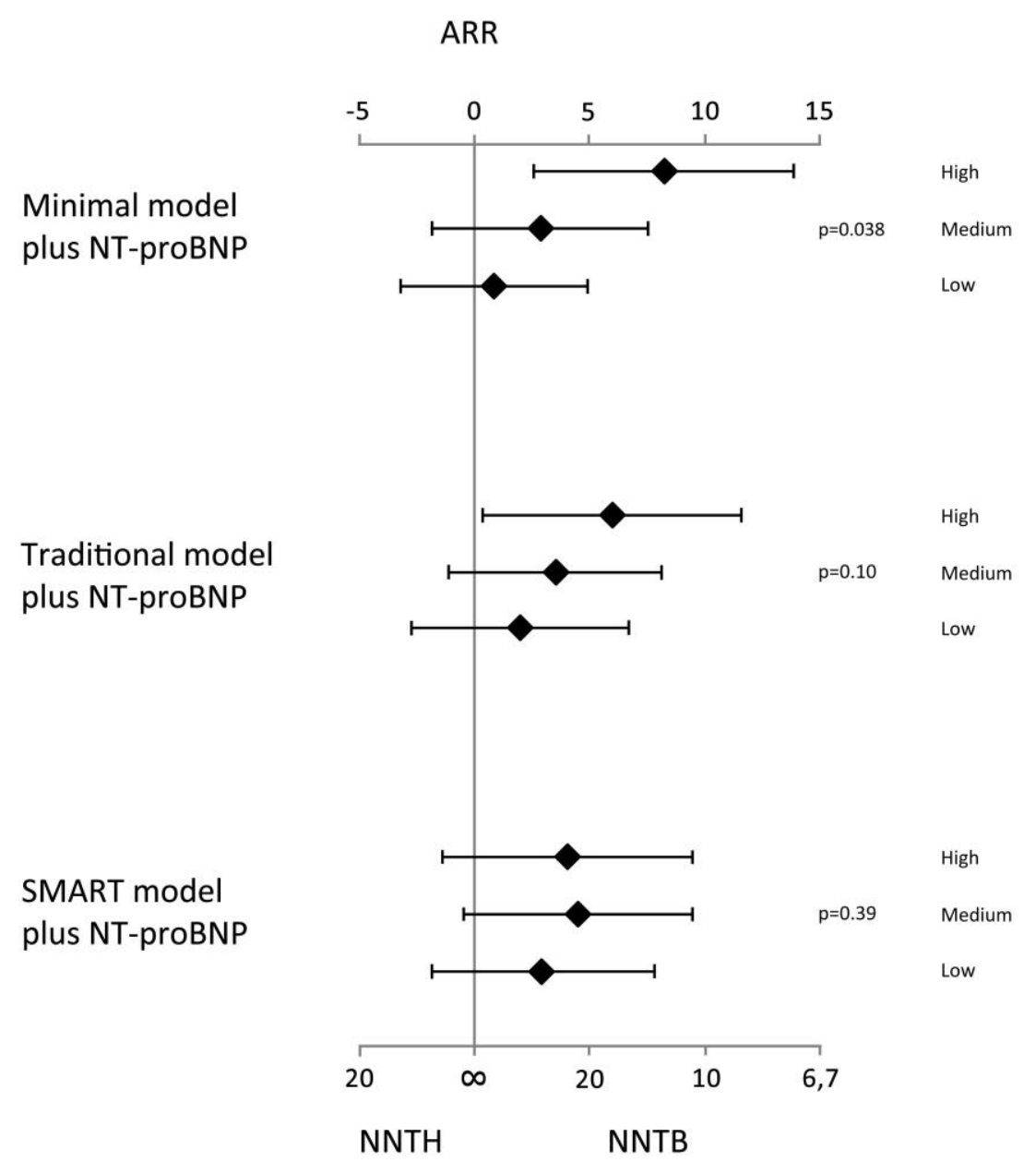

